\title{
Formation et évolution des exoplanètes géantes
}

Franck Selsis

Centre de Recherche Astronomique de Lyon, UMR 5574, École Normale Supérieure de Lyon

\begin{abstract}
Au cours de ces dernières années, l'observation des planètes géantes extrasolaires a passé le cap de la simple détection et permet désormais une caractérisation physico-chimique. Les modèles théoriques de formation, de composition et d'évolution de ces objets peuvent être confrontés à des observations de plus en plus riches, révélant la masse, le rayon, la température et certains constituants atmosphériques. Dans le même temps, le nombre de planètes détectées est devenu statistiquement significatif et impose des contraintes de plus en plus fortes sur les scénarios de formation des planètes, bouleversant les modèles qui s'étaient construits sur la base unique de notre système solaire. Le défi actuel est d'expliquer à la fois la diversité des systèmes planétaires rencontrés et la configuration du système solaire, et de répondre à la question suivante : la Terre fait-elle partie d'un système planétaire d'un type commun ou au contraire exceptionnel ?
\end{abstract}

Alors que l'on vient de fêter récemment le $10^{\mathrm{e}}$ anniversaire de la découverte par M. Mayor et D. Queloz de 51 Peg, la première planète extrasolaire orbitant autour d'une étoile de type solaire, le nombre des exoplanètes connues atteint désormais les 180. Ce nombre est devenu suffisamment élevé pour nous permettre de tirer quelques conclusions statistiques. Pour cela, il faut avant tout bien identifier les biais observationnels inhérents aux méthodes de détection. Celles-ci sont expliquées plus en détails qu'ici dans un récent article de Casoli et Encrenaz [1].

La très grande majorité de ces planètes sont découvertes par la mesure de la vitesse radiale ${ }^{(1)}$ de leur étoile, sur laquelle elles induisent une perturbation périodique. L'amplitude de cette perturbation permet de déterminer une valeur minimale du rapport entre la masse de la planète $\left(M_{P}\right)$ et celle de l'étoile $\left(M_{S}\right)$; l'inclinaison $i$ du système observé(2) n'étant pas connue, on ne peut mesurer que $\mathrm{M}_{\mathrm{P}} \times \sin (i) /\left(\mathrm{M}_{\mathrm{P}}+\mathrm{M}_{\mathrm{S}}\right)^{2 / 3}$. La connaissance de la masse de l'étoile est nécessaire pour déterminer $M_{p} \times \sin (i)$ et pour convertir la période en distance orbitale. La forme de la perturbation donne l'excentricité, une orbite circulaire donnant une perturbation sinusoïdale. Actuellement, on sait mesurer la vitesse d'une étoile à moins de $1 \mathrm{~m} / \mathrm{s}$, ce qui autorise la détection d'une réplique du couple Soleil-Jupiter (amplitude de la perturbation $=12 \mathrm{~m} / \mathrm{s}$ ) mais est encore insuffisant pour détecter une planète de mêmes masse et période que Saturne $(2,7 \mathrm{~m} / \mathrm{s})$. Outre cette contrainte sur la précision, la détection d'une planète par vitesse radiale implique des observations espacées sur une durée couvrant au moins une période orbitale, c'est-à-dire 11 ans pour un Jupiter et 29 ans pour un Saturne. II faudra donc attendre encore quelques années pour pouvoir révéler les systèmes planétaires similaires au système solaire.

Actuellement, et pour des étoiles de type solaire, on peut détecter des planètes jusqu'à environ 4 UA [Unité Astronomique = distance Terre-Soleil], si celles-ci sont suffisamment massives. II faut donc garder en tête que l'on détecte plus facilement les planètes à courte période (près de leur étoile) et massives que les planètes à longue période et peu massives. Une non-détection pour une étoile donnée ne permet donc pas de conclure à l'absence de planète, d'autant plus qu'un système donné peut très bien présenter une très faible inclinaison $i$ par rapport à l'observateur, ce qui peut rendre indétectable la perturbation projetée sur la ligne de visée.

(1) La vitesse radiale de l'étoile est la vitesse relative entre l'observateur et l'étoile observée. On la mesure par le décalage Doppler des raies spectrales de l'étoile. Cette vitesse est modulée par le mouvement de la Terre autour du Soleil, mais cette modulation est connue et facilement supprimée.

(2) L'inclinaison est l'angle entre la visée (direction Soleil-étoile) et l'axe de rotation du système planétaire. Lorsque l'observateur est dans le plan de l'orbite planétaire, l'inclinaison vaut $90^{\circ}$.
En moyenne, on trouve des planètes autour d'environ 10\% des étoiles observées. Cette abondance, ou plutôt cette probabilité de détection avec les moyens actuels, varie avec la composition chimique de l'étoile et peut atteindre $25 \%$ pour des étoiles de forte métallicité, c'est-à-dire riches en éléments plus lourds que He (figure 1). Environ 1\% des étoiles possèdent une planète plus massive que Saturne à moins de 0,1 UA. On appelle ces planètes, qui sont les plus faciles à détecter, des « Jupiters Chauds » ou «Pégasides ». Malgré un biais observationnel contraire, l'abondance des planètes décroît vers les masses élevées, chutant fortement au-dessus de 1-2 $M_{J}$ [masse de Jupiter], et augmente fortement lorsque l'on descend vers les faibles masses, ce qui tend à montrer que les planètes sub-saturniennes sont très abondantes. Les systèmes à plusieurs planètes sont assez communs (18 sont actuellement connus), et les excentricités des orbites au-delà de 0,1 UA sont étonnamment élevées (0,25 en moyenne) par rapport à celles qui sont rencontrées dans le système solaire.

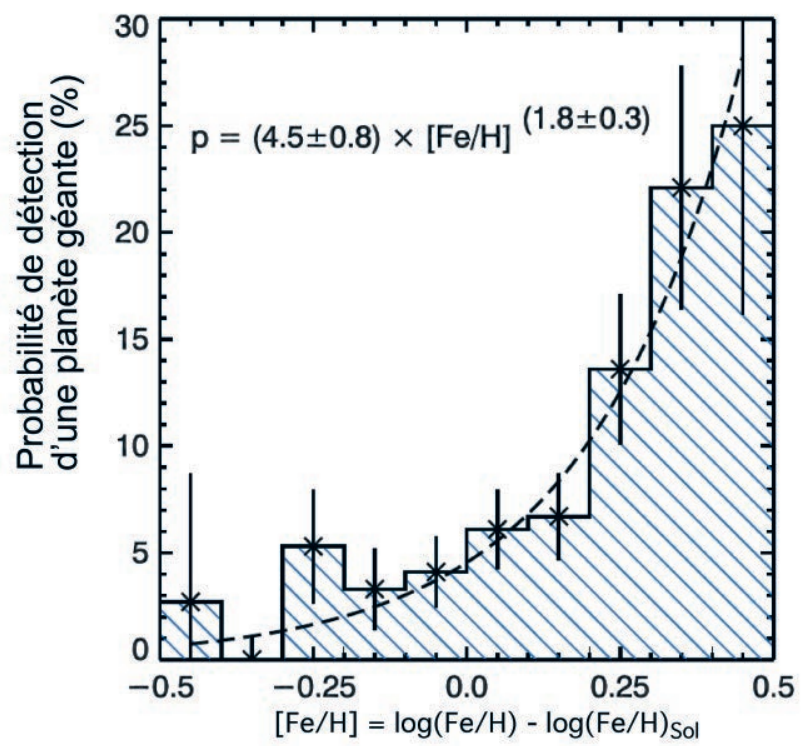

Figure 1 : Abondance de planètes géantes et métallicité. Les étoiles se distinguent par leur abondance en éléments lourds, appelée " métallicité » et représentée ici sur l'axe horizontal par l'abondance en fer relative à l'abondance solaire. Malgré le nombre encore restreint de planètes détectées ( 180), on peut déjà noter une forte corrélation entre la métallicité et la présence d'au moins une planète détectable. Pour les étoiles les plus " métalliques ", la probabilité de trouver une planète géante avec les moyens actuels est de 25\%. Ce lien n'a rien de surprenant si l'on considère que les planètes géantes se forment à partir de cœurs solides, constitués de ces éléments lourds (voir texte). D'après Valenti et al., Protostars \& Planets V (2005). 


\section{Les planètes qui transitent}

Lorsqu'une planète passe devant son étoile, la luminosité apparente du système est atténuée. Lorsqu'une planète de la taille de Jupiter éclipse une étoile de type solaire, cette diminution est d'environ $1 \%$ et dure de une à quelques heures (figure 2). Un tel événement est précieux car il permet de caractériser plus précisément la planète, mais il est aussi très rare. Pour un Jupiter à 5 UA d'une étoile solaire, la probabilité pour que l'orientation du système permette l'observation d'un transit est de $0,1 \%$. Imaginons que $10 \%$ des étoiles possèdent une réplique exacte de Jupiter et que l'on observe un champ d'étoiles en continu pendant une très longue durée : on détecterait alors 1 transit tous les 11 ans pour 10000 étoiles suivies ! Heureusement, la probabilité augmente fortement pour les périodes plus faibles et atteint $10 \%$ pour les Pégasides à 0,045 UA. De plus, dans le cas des Pégasides, le transit se reproduit tous les quelques jours. II devient donc intéressant de rechercher systématiquement les transits éventuels pour les Pégasides déjà détectées par vitesse ; c'est ainsi que trois planètes à transits ont été identifiées, dont la première, HD209458b. Sachant qu'environ 1\% des étoiles possèdent une Pégaside, l'observation pendant quelques semaines d'un champ de 10000 étoiles doit révéler environ dix planètes. Actuellement, on connaît neuf planètes qui tran-
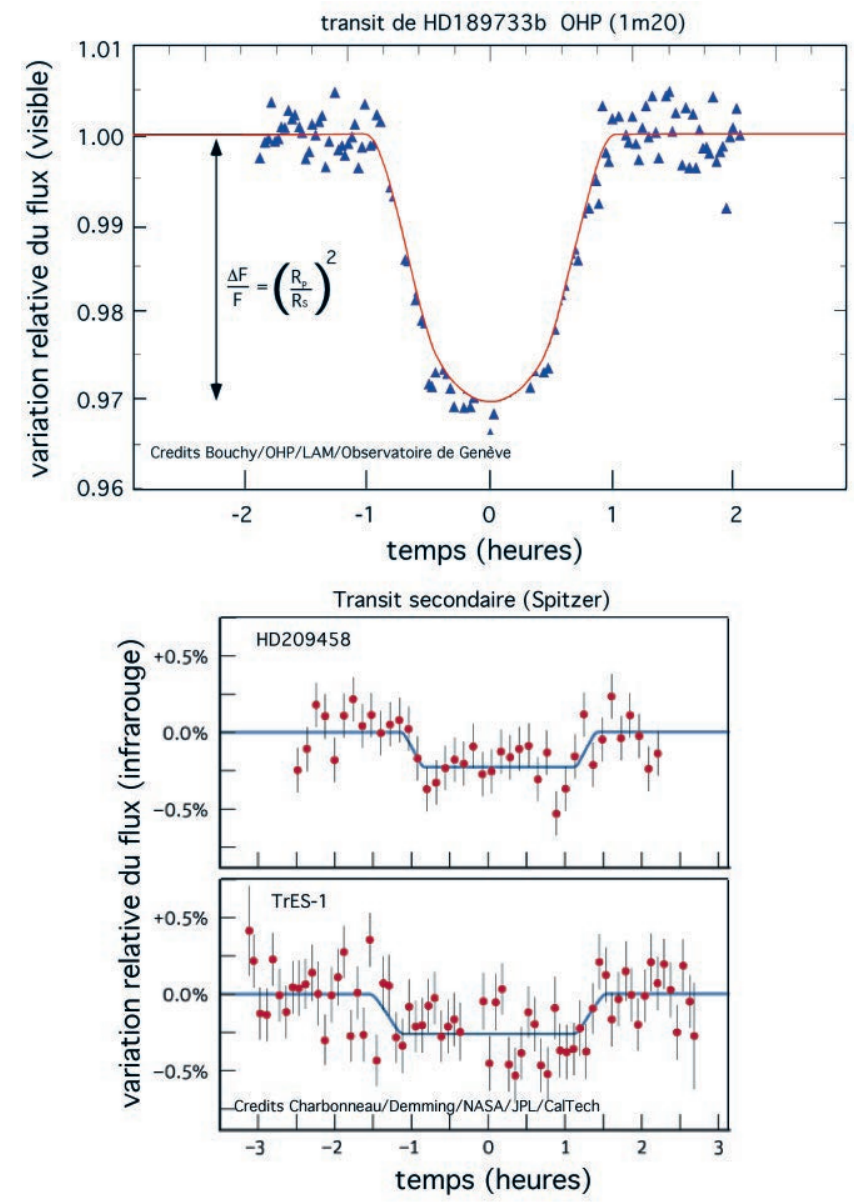

Figure 2 : Courbes de lumière lors de transits primaires et secondaires. La figure d'en haut montre la variation de luminosité apparente de l'étoile HD189733 en lumière visible (enregistrée au télescope de $1 \mathrm{~m} 20$ de l'Observatoire de Haute-Provence) lorsqu'elle est partiellement éclipsée par sa planète. La profondeur du transit, $\Delta F / F$, est directement reliée au rapport entre le rayon de la planète, $R_{p}$, et celui de l'étoile, $R_{S}$. Le fond arrondi de la courbe est dû à l'assombrissement centre-bord de l'étoile. Les graphes d'en bas montrent la courbe de lumière, infrarouge cette fois, de deux étoiles, HD209458 et TrES-1, mesurée lorsque la planète transite derrière l'étoile. Cet effet est beaucoup plus faible que le transit primaire et n'a pu être observé que très récemment grâce au télescope spatial Spitzer [2, 3]. sitent devant leur étoile. Leur période varie de 1,2 à 4 jours. La majorité d'entre elles ont été détectées par le transit lui-même et non par vitesse radiale.

Le transit permet de déterminer le rapport entre le rayon de l'étoile et celui de la planète. II lève aussi l'ambiguïté sur l'inclinaison $i$ du système et donc sur la masse de la planète lorsque celle-ci est aussi détectée par vitesse radiale, ce qui est le cas pour toutes les planètes à transit connues. Les précisions sur le rayon et la masse de la planète dépendent de la connaissance que l'on a de l'étoile parente. Pour les étoiles proches, comme HD209458 ou HD189733, dont la planète a été initialement détectée par vitesse radiale, l'étoile parente est bien connue et la précision est excellente. Pour les détections issues d'observations systématiques de champs d'étoiles lointaines (comme les étoiles OGLE), la détermination des paramètres physiques est beaucoup moins précise.

Outre la détermination du rayon et de l'inclinaison, les transits permettent de pousser encore plus loin la caractérisation. Tout d'abord, pour une planète possédant une atmosphère, le rayon planétaire mesuré par l'atténuation du flux stellaire lors du transit dépend de la longueur d'onde à laquelle ce flux est mesuré. En effet, si un composé présent dans la très haute atmosphère absorbe le rayonnement à $\lambda_{1}$ alors que l'atmosphère est transparente à $\lambda_{2}$ jusque dans ses couches très denses, on va avoir $R\left(\lambda_{2}\right)<R\left(\lambda_{1}\right)$. Donc, si l'on est capable de mesurer correctement la courbe de lumière et la profondeur du transit dans deux bandes spectrales fines, on peut détecter des constituants de l'atmosphère. C'est ainsi que l'on a identifié du sodium, de l'hydrogène [4] et peut-être du carbone et de l'oxygène dans la haute atmosphère de HD209458b.

Enfin, on est désormais capable de détecter l'émission thermique de la planète lors du transit secondaire (figure 2), juste avant et juste après que la planète passe derrière son étoile. Si l'on est capable de voir la diminution de l'émission infrarouge du système non résolu étoile+planète qui survient à ce moment, on peut estimer la fraction du flux en provenance de la planète. Puisque l'on connaît le rayon de la planète, on peut dès lors déterminer la température de brillance de l'objet et un albédo moyen, c'est-à-dire le pouvoir réfléchissant de la planète, qui dépend de la composition de l'atmosphère.

Le télescope Spitzer a permis d'effectuer ce type de mesure pour trois des planètes à transits, dans plusieurs bandes spectrales différentes, ce qui permet désormais de comparer avec les spectres théoriques que l'on calcule pour les Pégasides. Ces mesures donnent une information à très basse résolution, et affectée par un bruit important, qui ne permet d'obtenir qu'une information limitée. Toutefois, des observations de meilleure qualité pourront être réalisées dans le futur avec l'observatoire spatial JWST. Les spectres obtenus devraient permettre de détecter la présence des constituants atmosphériques prédits par les modèles théoriques (essentiellement $\mathrm{H}_{2} \mathrm{O}$ et $\mathrm{CO}$ ), l'effet de nuages, et le contraste de température entre les hémisphères jour et nuit de ces planètes.

\section{Formation des planètes géantes}

Deux scénarios sont invoqués pour la formation des planètes géantes. Dans les deux cas, les planètes naissent dans le disque circumstellaire de gaz et de poussière qui se forme lors de l'effondrement d'un nuage interstellaire en une protoétoile. Dans ce disque dit protoplanétaire, les poussières s'agglutinent, en quelques milliers d'années, en une population de planétésimaux, dont la taille dépasse le $\mathrm{km}$. Dans le modèle dit de "core accretion" de Pollack et al. [5], un cœur solide de glace et de roche se forme dans un premier temps par collisions de planétésimaux dans le disque protoplanétaire. Cette phase est similaire à la formation des planètes de type terrestre. Quand la gravité de ce cœur devient suffisamment élevée pour que la vitesse de libération dépasse la vitesse thermique moyenne du gaz environnant, la planète commence à 
accréter une enveloppe gazeuse ; c'est la phase 2. Enfin, lorsque ce cœur et son atmosphère atteignent une masse critique de l'ordre de $10 \mathrm{M}_{\mathrm{T}}$ (masse terrestre), le disque s'effondre localement sur la protoplanète qui acquiert ainsi une enveloppe massive de gaz. C'est dans cette $3^{e}$ phase que la planète atteint sa masse finale ( $318 \mathrm{M}_{\mathrm{T}}$ dans le cas de Jupiter). L'accrétion prend fin lorsque la masse de la planète devient telle qu'elle ouvre un sillon dans le disque et s'isole partiellement du gaz du disque ou lorsque le disque de gaz est totalement dissipé.

Un scénario alternatif, défendu en particulier par Boss [6], invoque un effondrement local du disque protoplanétaire, lié à des instabilités gravitationnelles, et conduisant à la formation d'une protoplanète de composition « solaire ". Une fois formée, cette planète peut s'enrichir en éléments lourds en capturant des planétésimaux et, éventuellement, former un cœur solide. Toutefois, contrairement au modèle d'accrétion sur un cœur solide, la formation de planètes géantes par instabilité gravitationnelle n'explique pas la forte corrélation que l'on observe entre la présence de planètes géantes et la métallicité de l'étoile centrale, c'est-à-dire l'abondance des éléments plus lourds que $\mathrm{He}$ (essentiellement $\mathrm{O}, \mathrm{C}$ et $\mathrm{Fe}$ ). Dans l'échantillon des étoiles observées dans le cadre de la recherche de planètes, environ $5 \%$ des étoiles ayant une métallicité solaire possèdent une planète détectable à moins de $3 \mathrm{UA}$. Cette abondance (ou probabilité de détection) passe à $25 \%$ pour une métallicité trois fois solaire (figure 1). Cette relation s'explique dans le modèle de l'accrétion sur un cœur, qui dépend naturellement de la densité surfacique de matériel solide présent dans le disque et disponible pour la formation du cœur. De plus, le modèle d'instabilité gravitationnelle ne permet pas d'expliquer les planètes géantes glacées de type Uranus et Neptune.

\section{Un problème majeur : le manque de temps et de solides}

Jusqu'à récemment, et bien qu'étant considérée comme le modèle " standard " de la formation des planètes géantes, l'accrétion sur un corps solide posait toutefois un problème sérieux : la durée de formation d'un Jupiter par ce mécanisme excède largement la durée de vie des disques protoplanétaires observés autour d'étoiles jeunes. En effet, si près de $100 \%$ des étoiles les plus jeunes montrent les signes observationnels de la présence d'un disque protoplanétaire, la fraction des étoiles possédant un disque tombe à $\sim 0 \%$ en moins de $10 \mathrm{Ma}$ [millions d'années]. Les mécanismes de dissipation des disques sont mal connus, certainement multiples et sans doute variables d'un système à l'autre. En premier lieu, le disque est accrété sur l'étoile. Une partie importante de sa masse disparaît sans doute dans la formation des planètes géantes. Il est de plus photoévaporé par le rayonnement $X$ et UV de l'étoile centrale et d'étoiles très massives formées dans la même région. Enfin, il est aussi dispersé par le vent stellaire et subit des interactions gravitationnelles avec les étoiles voisines.

Si l'on considère une formation planétaire locale (la planète n'accrète des planétésimaux que dans une zone restreinte du disque protoplanétaire), le temps nécessaire à la formation d'un cœur de masse critique, permettant d'initier la formation rapide d'une géante gazeuse, s'avère bien supérieur à la durée de vie observée des disques. Le désaccord est suffisamment important pour être significatif : pour rendre le modèle d'accrétion de Pollack compatible avec ce temps de dissipation des disques, il faudrait donner aux paramètres du modèle (densité surfacique de planétésimaux, opacité du disque) des valeurs irréalistes. Cette incompatibilité a relancé pour quelque temps l'hypothèse de la formation par instabilité gravitationnelle, qui se déroule beaucoup plus rapidement : dans les simulations numériques, il faut en effet moins de 1000 ans pour former une condensation autogravitante de $1 \mathrm{M}_{\mathrm{J}}$ (masse de Jupiter).
Avant la découverte des planètes géantes extrasolaires, la configuration du système solaire, avec des petites planètes telluriques dans la partie interne et des planètes géantes dans la partie externe, s'insérait bien dans la théorie d'accrétion sur cœur solide. En effet, il existe dans le disque protoplanétaire une distance limite au-delà de laquelle l'eau n'existe que sous forme de glace. La densité surfacique de solide augmente considérablement lorsque l'on franchit cette ligne de gel en s'éloignant de l'étoile centrale. Ceci peut expliquer quantitativement la formation de planètes rocheuses de masse $\sim 1 \mathrm{M}_{\mathrm{T}}$ à l'intérieur de la ligne de gel, et de cœurs de roche et de glace atteignant $10 \mathrm{M}_{\mathrm{T}}$ au-delà. La découverte des exoplanètes, et en particulier des Pégasides, qui ont des périodes orbitales de quelques jours, a fortement bouleversé cette vision bien organisée des choses. Comment former une planète de $\sim 1 \mathrm{M}_{\jmath}$ à $0,05 \mathrm{UA}$, c'est-à-dire 100 fois plus près de son étoile que Jupiter du Soleil ? La position précise de la ligne de gel dans un disque protoplanétaire est mal connue et évolue avec le temps mais, autour d'une étoile solaire, elle se situe toujours au-delà de l'orbite terrestre et ne peut donc pas expliquer l'existence des Pégasides.

\section{La migration au secours de la formation planétaire}

Au cours des 10 ans écoulés depuis la découverte de $51 \mathrm{Peg}$, on s'est peu à peu rendu compte de l'importance de la migration planétaire. Une planète qui se forme dans un disque de gaz migre vers son étoile en raison de plusieurs mécanismes désormais bien identifiés [7]. Le cœur des Pégasides s'est vraisemblablement formé au-delà de la ligne de gel, à des distances orbitales similaires à celle de Jupiter, puis la planète en formation a migré vers son étoile pour s'arrêter près du bord interne du disque. Non seulement la migration explique l'existence des Pégasides, mais elle réhabilite aussi le modèle d'accrétion sur cœur solide, en l'accélérant notablement. Dans le modèle original où la formation de la planète a lieu localement, la croissance du cœur par accrétion de planétésimaux s'arrête vite, car la planète vide sa zone de formation des solides qui s'y trouvaient, et c'est par l'accrétion lente de gaz que la protoplanète doit atteindre sa masse critique qui déclenche l'effondrement. Alibert et al. [8] ont récemment montré que la migration du cœur pendant sa croissance lui permettait d'accréter beaucoup plus de planétésimaux et d'atteindre ainsi sa taille critique dans un temps de l'ordre du million d'années, compatible avec la durée de vie des disques.

Si le rôle de la migration dans la formation de Jupiter et des exoplanètes géantes semble clairement établi, le phénomène est quantitativement difficile à modéliser, et plusieurs questions restent à élucider. Quel mécanisme, par exemple, stoppe la migration vers 0,04 UA, distance orbitale autour de laquelle semblent s'accumuler les Pégasides ? Cette distance correspond-elle au bord interne du disque qui le sépare d'une cavité creusée par l'étoile ? Des planètes tombent-elles sur leur étoile ou s'arrêtent-elles forcément au niveau de cette limite ? Pourquoi la migration de Jupiter s'est-elle arrêtée à 5 UA alors que les Pégasides ont continué leur migration jusqu'aux abords immédiats de leur étoile ? Nous n'avons pas à l'heure actuelle de réponses précises à ces questions, mais plusieurs pistes s'offrent à nous pour comprendre les destins différents de Jupiter et des Pégasides.

Tout d'abord, les disques planétaires ont des masses différentes et sont soumis à des contraintes extérieures qui varient selon la densité locale d'étoiles. Les temps caractéristiques de dissipation des disques étant similaires aux temps de formation des planètes géantes et probablement aux temps de migration, il est normal que ces conditions dynamiques et radiatives externes variables aboutissent à une diversité de systèmes planétaires. Les disques les plus massifs et les plus isolés ont sans doute plus de chance de produire des Pégasides que des disques où la formation planétaire est interrompue par la dissipation du gaz disponible. 
II est aussi possible que les planètes géantes se forment préférentiellement par paires, comme notre couple JupiterSaturne. Lorsqu'un Jupiter se forme, il ouvre un sillon dans le disque et migre vers l'intérieur à mesure que le disque s'accrète sur l'étoile. Sur le bord externe de ce sillon, il y a accumulation de planétésimaux, ce qui favorise la formation d'un second cœur massif d'accrétion et d'une seconde planète. En ouvrant un sillon commun, le couple de planètes migrerait beaucoup plus lentement vers son étoile et pourrait rester proche de sa zone de formation jusqu'à la dissipation du disque et l'arrêt de la migration. Ce scénario de formation de couples Jupiter-Saturne est pour l'instant spéculatif et ne repose que sur des éléments théoriques qualitatifs. Seule l'observation future de planètes extrasolaires à plus longue période pourra nous révéler si le système solaire est de configuration commune ou, au contraire, très rare. À l'heure actuelle, moins de $10 \%$ des étoiles étudiées nous révèlent un compagnon planétaire détectable, mais si les $90 \%$ restants abritaient des répliques exactes du système solaire, nous n'en saurions toujours rien.

\section{Structure, composition et évolution}

L'évolution d'une planète géante durant la première centaine de millions d'années (Ma) qui suit sa formation est mal connue, car il est difficile de contraindre le rayon initial de l'objet. Toutefois, après $100 \mathrm{Ma}$, la structure ne dépend plus de cet état initial et l'on peut modéliser l'évolution du rayon et de la luminosité d'une planète de masse donnée. Ces modèles donnent de très bons résultats pour les étoiles de faible masse et les naines brunes. Appliqués aux exoplanètes, on s'est aperçu que ces mêmes modèles sous-estiment nettement le rayon observé des planètes à transit si l'on ne prend pas en compte l'effet de l'irradiation par l'étoile centrale sur la structure et l'évolution de la planète. Une planète soumise à une forte irradiation se refroidit en effet moins efficacement, ce qui ralentit sa contraction. À un âge donné, une planète non irradiée sera donc plus compacte qu'une planète irradiée de même masse et de même composition. Une fois l'irradiation incluse dans la modélisation, l'accord s'avère satisfaisant [9], sauf pour une planète, HD209458b, dont le rayon est inexplicablement élevé.

Les figures 3 et 4 présentent la comparaison des modèles de structure et d'évolution avec les masses et rayons déduits des observations de transits planétaires. Les zones en jaune (irradiation incluse) et orange (sans irradiation stellaire) délimitent les valeurs possibles de la masse et du rayon pour une planète de composition solaire (sans cœur solide et non enrichie en éléments lourds) dont l'âge est compris entre 1 et 8 Ga [milliards d'années] (figure 3) et dont la masse est comprise entre 0,5 et $2 M_{J}$ (figure 4). Pour une masse donnée, le rayon de la planète sera toujours plus faible si la planète possède un cœur solide ou est enrichie en éléments lourds. Il est donc toujours possible de trouver une planète en dessous des zones jaune et orange dans ces diagrammes. Par exemple, la masse de la planète HD149026 est dominée par les éléments lourds autres que $\mathrm{H}$ et $\mathrm{He}$, et possède vraisemblablement un important cœur de roche. Cela semble cohérent avec le fait que son étoile centrale a une métallicité fortement sursolaire.

Toutes les planètes à transits sont donc compatibles avec les modèles théoriques, sauf HD209458b qui fut la première découverte et qui est trop peu dense par rapport à ce que l'on prédit. Pour maintenir ce rayon très élevé, une quantité énorme d'énergie doit être dissipée dans les couches convectives profondes de la planète. Le mécanisme à l'origine de cette dissipation n'est pas identifié. II pourrait s'agir de mouvements dynamiques liés au fort gradient thermique entre le côté jour de la planète et le côté nuit, capables de convertir une fraction de l'irradiation stellaire (moins de $1 \%$ suffit) en chaleur. Cela pourrait aussi venir d'effets de marées déformant la planète et dissipant de l'énergie dans la structure

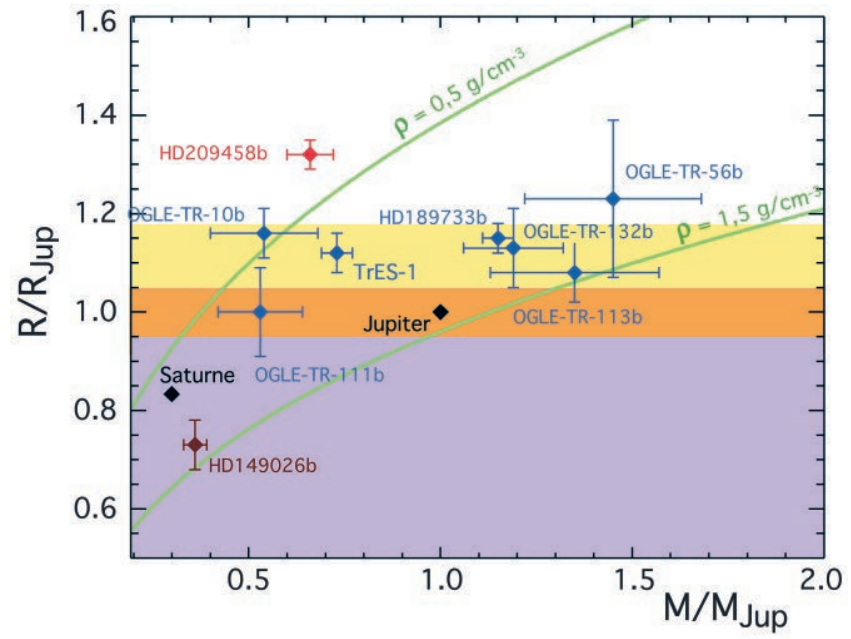

Figure 3 : Masses et rayons des neuf Pégasides produisant des transits. Les zones jaune et orange délimitent l'intervalle de rayon prédit pour des planètes de composition solaire (sans cceur de roche), pour des âges allant de 1 à 8 milliards d'années pour la gamme d'irradiation de ces neuf planètes (jaune) et sans irradiation (orange). On note que le rayon est peu dépendant de la masse, ce qui est une manifestation frappante de la mécanique quantique, puisque c'est ici la pression de dégénérescence des électrons qui s'oppose à la gravité. La zone violette correspond à des planètes très enrichies en éléments lourds. La très faible densité de HD209458b (une planète au moins aussi vieille que le système solaire) ne peut être expliquée avec les modèles actuels. À l'opposé, les dimensions réduites de HD149026b impliquent une composition très enrichie en éléments lourds (plus de $60 \%$ de la masse). Pour indication, les traits verts suivent des densités constantes.

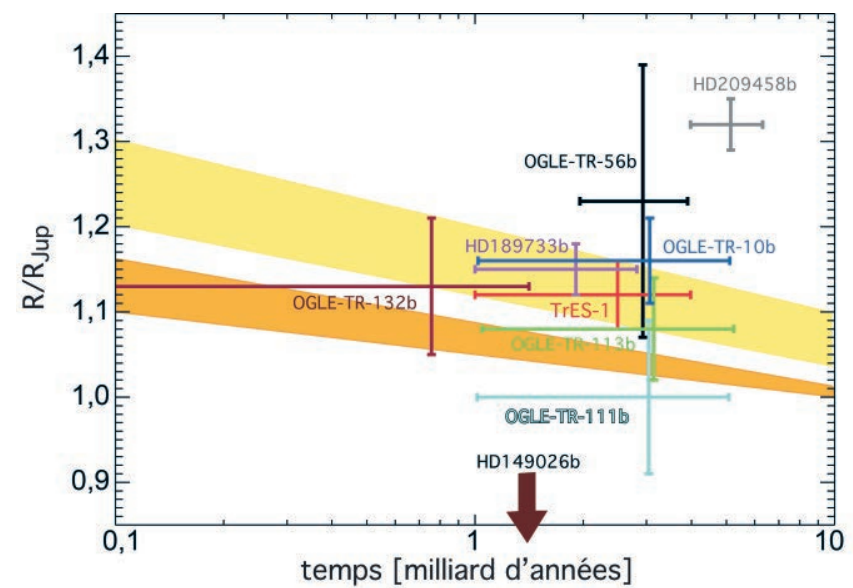

Figure 4 : Évolution du rayon des exoplanètes géantes. Les régions jaune et orange délimitent le domaine d'évolution théorique pour des planètes géantes gazeuses de composition solaire, et pour les masses observées des neuf planètes à transit connues. Comme dans la figure 3 , la région jaune correspond à un calcul d'évolution incluant l'irradiation de l'étoile centrale, tandis que la zone orange représente les cas non irradiés. Un enrichissement en éléments lourds produit des planètes plus compactes et décalerait les courbes d'évolution vers le bas. HD149026b n'apparait pas sur ce diagramme et peut difficilement être qualifiée de "géante gazeuse ", puisqu'elle doit être constituée à plus de 60\% d'éléments plus lourds que He, probablement concentrés en un énorme cœur de roche.

même de la planète. Une autre hypothèse invoquée est une perte de masse violente, liée à l'irradiation $X$ et UV de l'étoile et à l'érosion par le vent stellaire. Mais, pour cela, il faudrait que l'évaporation soit suffisamment intense pour que l'évolution de la masse de la planète se fasse sur des échelles de temps plus courtes que sa contraction. Ce cas est possible, mais vraisemblablement pas pour HD209458b, car une planète dans un tel régime serait condamnée à très brève échéance à l'évaporation totale de son enveloppe gazeuse, et la probabilité d'observer une planète âgée de plus de $4 \mathrm{Ga}$ dans cette phase semble minime. Logiquement, le mécanisme en action sur HD209458b devrait agir aussi sur les autres 
Pégasides. II est donc possible que les autres planètes à transits possèdent des cœurs très massifs, mais que ce même phénomène augmente leur rayon et le fasse coïncider avec les valeurs obtenues théoriquement pour des planètes de composition solaire (sans coeur massif). Sur les figures 3 et 4, l'accord entre les rayons observés et les courbes en pointillés pourrait donc être trompeur.

\section{Évaporation}

La majorité des planètes détectées orbitent à de faibles distances et subissent donc une irradiation très forte de leur étoile. Les Pégasides, dont la période orbitale est de quelques jours seulement, reçoivent un flux de rayonnement $\sim 500$ et $\sim 10000$ fois supérieur à ceux reçus par la Terre et par Jupiter, respectivement. Nous avons déjà vu que cette irradiation modifiait la structure interne et le rayon par rapport à une planète faiblement irradiée.

Un second effet est dû à l'irradiation par le rayonnement $X$ et extrême UV (que nous appelons communément XUV et qui couvre le domaine spectral de 0,1 à $100 \mathrm{~nm}$ ) : il s'agit de l'érosion atmosphérique, ou évaporation. Les couches les plus externes de l'atmosphère sont chauffées par le rayonnement XUV et la température peut devenir suffisante pour qu'une fraction de l'hydrogène s'échappe de l'attraction gravitationnelle de la planète. Cet échappement est rendu plus efficace encore par l'ionisation du gaz et l'interaction avec le vent stellaire, très dense et rapide aux faibles distances orbitales considérées. Les simulations montrent que la combinaison de l'expansion des couches externes de l'atmosphère par le chauffage XUV et de l'érosion par le vent stellaire peuvent entraîner une perte de masse très importante pouvant théoriquement modifier la masse totale de la planète au cours de son existence. Vidal-Madjar et al. [4] ont mesuré une absorption dans la raie Lyman- $\alpha$ (121,6 nm) de l'étoile HD 209458 qui se produit à chaque transit de la planète et qui peut être interprétée comme une couronne d'hydrogène en échappement. Cette absorption ne permet toutefois que de fixer une limite inférieure sur la perte de masse, et ce flux minimum n'aurait qu'un effet mineur sur l'évolution de la planète. II est aussi possible que tout ou partie de cette couronne d'hydrogène associée à HD209458b soit dû à la neutralisation du vent stellaire au contact de la magnétosphère de la planète. II est difficile d'estimer le taux de perte des Pégasides, car le mécanisme d'échappement couple des effets thermiques (chauffage XUV) et non thermiques (interaction avec le vent solaire) qui ne peuvent être séparés, l'expansion thermique de la haute atmosphère exposant le gaz atmosphérique au vent stellaire [10].

Pour les planètes à faible distance orbitale mais moins massives que les Pégasides, c'est-à-dire des "Saturnes et Neptunes chauds ", les effets de l'évaporation sont certainement considérables. Ces planètes, que l'on commence à détecter par vitesse radiale, peuvent perdre la quasi-totalité de leur enveloppe gazeuse, comme le montre la figure 5. La planète-test considérée sur ce graphe a des caractéristiques comparables [Msin(i), distance orbitale a] à celles de certaines des planètes dernièrement détectées par vitesse radiale : Gliese 876 d [7 $\left.\mathrm{M}_{\text {Terre }}, 0,02 \mathrm{UA}\right], \mathrm{HD} 160691$ d [14 $\left.\mathrm{M}_{\text {Terre }}, 0,09 \mathrm{UA}\right], 55$ Cnc e [14 M $\left.\mathrm{M}_{\text {Terre }}, 0,04 \mathrm{UA}\right]$, Gl 581 b [18 $\mathrm{M}_{\text {Terre }}, 0,04$ UA]...

Si le cœur de ces planètes est constitué en partie de glace (n'oublions pas que dans le scénario de formation actuel, ces objets se forment dans la partie externe et froide du disque protoplanétaire), l'érosion peut toucher la partie solide. À mesure que l'atmosphère s'échappe, celle-ci est alimentée par la vaporisation des glaces. Suivant la masse initiale de glace et de gaz dont dispose la planète, sa distance orbitale et sa masse, ce régime peut continuer indéfiniment ou au contraire ne laisser rapidement qu'un cœur rocheux résiduel.

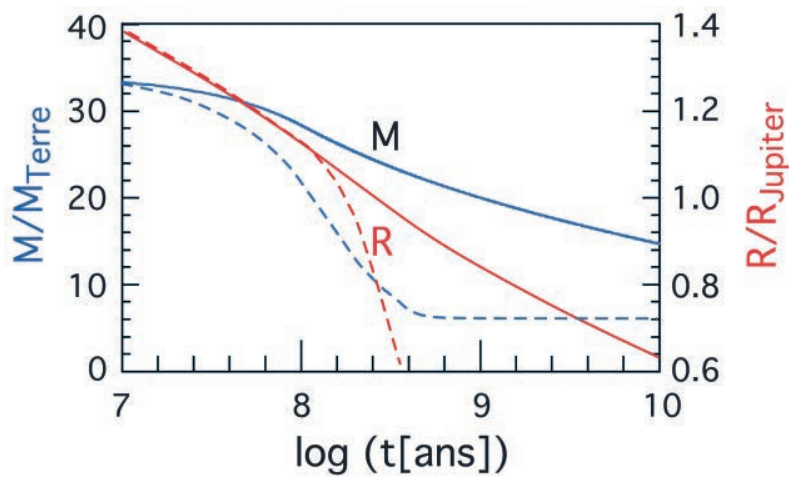

Figure 5 : Évolution et évaporation. Ce graphe illustre le destin possible d'une planète géante ayant migré près de son étoile durant sa formation. Dans cette simulation, une planète de $33 M_{T}$ composée d'un cœur solide de $6 M_{T}$ et d'une enveloppe de composition solaire est placée à 0,1 UA de son étoile centrale (de type solaire). Les courbes représentent l'évolution de la masse (en bleu) et du rayon (en rouge) de la planète, pour des taux d'échappement qui diffèrent d'un facteur 2 (traits pleins et traits pointillés - l'incertitude réelle sur ce taux est beaucoup plus importante). À cette distance, une planète d'une masse supérieure à celle de Saturne (95 $\left.M_{T}\right)$ serait marginalement affectée, mais ici notre planète-test perd au minimum la moitié de sa masse au cours de son existence. À plus faible distance orbitale, les effets seraient plus impressionnants encore. D'après Baraffe et al. [11].

\section{Perspectives}

L'observation des exoplanètes nous a révélé un bestiaire étonnant de planètes qui n'ont pas d'équivalent dans le système solaire. Passée la surprise initiale de trouver des planètes géantes à quelques rayons stellaires de leur étoile, nous comprenons de mieux en mieux comment de tels objets ont pu se former et quelle peut être leur évolution, bien que les détails quantitatifs nous échappent encore. Dans quelques mois, le satellite français Corot devrait nous en apprendre plus sur les planètes à courte période orbitale, dont l'évolution est soumise à la prodigieuse irradiation de leur étoile parente. Corot pourrait aussi nous offrir la première détection d'une planète tellurique de quelques masses terrestres.

Une question fascinante restera en suspens quelques années encore : le système solaire est-il un système planétaire d'un type commun dans la galaxie ou fait-il au contraire office d'exception? Un « système solaire », c'est avant tout un Soleil plus un Jupiter. Les exo-Jupiters à plus de 5 UA de leur étoile devraient être détectés par vitesse radiale dans les 5-10 années à venir. Mais ce qui fait à nos yeux l'unicité du système solaire, c'est bien évidemment la Terre. Pour dévoiler les exo-Terres, il faudra se doter d'observatoires spatiaux de nouvelle génération, tel que l'interféromètre Darwin, dont le but est de détecter directement et d'étudier spectroscopiquement les autres Terres. Ce projet ambitieux sera l'aboutissement de la recherche d'exoplanètes dans le voisinage solaire, et le premier pas vers la recherche d'autres mondes abritant la Vie.

\section{Références}

[1] Casoli et Encrenaz, 2005, numéro commun du BUP ( ${ }^{\circ}$ 875) et du Bulletin de la SFP (hors-série), pp. 117-123.

[2] Charbonneau et al., 2005, The Astrophysical Journal 626-1, 523529. http://lanl.arxiv.org/abs/astro-ph/0503457

[3] Deming et al., 2005, Nature 434, 740-743. http://lanl.arxiv.org/abs/astro-ph/0503554

[4] Vidal-Madjar et al., 2003, Nature 422, 143-146.

[5] Pollack et al., 1996, Icarus 124-1, 62-85.

[6] Boss, 1997, Science 276, 1836-1839.

[7] Terquem et Papaloizou, La Recherche 348 (déc. 2001), 36-39.

[8] Alibert et al., 2004, Astronomy and Astrophysics 417, L25-L28. http://lanl.arxiv.org/abs/astro-ph/0403574

[9] Chabrier et al., 2004, The Astrophysical Journal 603, L53-L56. http://lanl.arxiv.org/abs/astro-ph/0401487

[10] Kodatchenko et al., Planetary \& Space Science, à paraître.

[11] Baraffe et al., 2006, Astronomy and Astrophysics, à paraître. http://lanl.arxiv.org/abs/astro-ph/0512091 